\title{
MINORS IN WEIGHTED GRAPHS
}

\author{
CEZAR JOIŢA ${ }^{凶}$ and DANIELA JOIŢA
}

\author{
(Received 15 August 2007)
}

\begin{abstract}
We define the notion of minor for weighted graphs. We prove that with this minor relation, the set of weighted graphs is directed. We also prove that, given any two weights on a connected graph with the same total weight, we can transform one into the other using a sequence of edge subdivisions and edge contractions.
\end{abstract}

2000 Mathematics subject classification: 05C83.

Keywords and phrases: graph minors, weighted graphs.

\section{Introduction}

The notion of minor is a central one in graph theory. Of course, the study of minorclosed classes of graphs culminated with the proof by Robertson and Seymour [2] of Wagner's conjecture. For an excellent survey, see Lovász's paper [1].

In this paper we define the notion of minor for weighted graphs. Since, given any connected network, it is not desirable to disconnect it, we shall work only with connected graphs. And since, when defining a weight, one has to choose a unit, we can restrict ourselves to graphs of total weight 1 . This is the same as identifying two weights if one of them is a multiple of the other. The two operations used to define a minor, for weighted graphs, are the two standard ones: edge contraction and edge deletion. One has to define what happens to the flow through a deleted or contracted edge. The definition that we adopt here is, we think, the most natural one. Namely, the flow is distributed proportionally to the adjacent edges. It can be seen easily that with this definition, Wagner's conjecture does not hold.

Our first theorem states that with this minor relation, the set of weighted graphs is directed. This is not obvious since a subgraph is no longer a minor. In fact, we prove that for any two weighted graphs, we can find another one which has them both as minors and subgraphs.

In the second part of the paper we show that, given any two weights on a connected graph with the same total weight, we can transform one into the other using a sequence

The first named author was supported by a Marie Curie International Reintegration Grant no. 01302.

(C) 2008 Australian Mathematical Society 0004-9727/08 \$A2.00+0.00 
of edge subdivisions and edge contractions. However, in general, we cannot perform all the edge subdivisions at the beginning and then the edge contractions. All proofs are constructive.

\section{Results}

In what follows we shall work with simple (without loops or multiple edges), undirected and connected graphs. By a 'weighted graph' we mean a pair $(G, c)$ where $G$ is a graph, whose set of vertices and edges will be denoted by $V(G)$ and $E(G)$ respectively, and $c: E(G) \rightarrow(0, \infty)$ is the weight function. If $H$ is a subgraph of $G$, we denote by $c(H)$ the total weight of $H$, that is, $c(H)=\sum_{e \in E(H)} c(e)$. If $A$ is a vertex of $G$, we denote by $c(A)$ the sum of the weights of all edges at $A$.

Definition 1. Given two weighted graphs $\left(G_{1}, c_{1}\right)$ and $\left(G_{2}, c_{2}\right)$, we say that they are equivalent and we write $\left(G_{1}, c_{1}\right) \sim\left(G_{2}, c_{2}\right)$, if $\left(G_{1},\left[1 /\left(c_{1}\left(G_{1}\right)\right)\right] c_{1}\right)$ and $\left(G_{2},\left[1 /\left(c_{2}\left(G_{2}\right)\right)\right] c_{2}\right)$ are isomorphic. We denote by $[(G, c)]$ the equivalence class of $(G, c)$.

DEFINITION 2. For a weighted graph $(G, c)$ we define the following two operations.

(1) Deleting an edge. This operation is allowed only if the resulting graph is connected, that is, the edge is not a bridge. The weight of the deleted edge is redistributed proportionally to the adjacent edges. This means that if the deleted edge is $e$ and its adjacent edges are $e_{1}, e_{2}, \ldots, e_{k}$, then their new weights will be

$$
c_{e}\left(e_{j}\right)=c\left(e_{j}\right)+\frac{c(e) c\left(e_{j}\right)}{c\left(e_{1}\right)+c\left(e_{2}\right)+\cdots+c\left(e_{k}\right)} .
$$

The weight of an edge not adjacent to $e$ remains unchanged. The resulted graph is denoted by $\left(G-e, c_{e}\right)$.

(2) Contracting an edge $e$. The new weights are defined as follows:

(a) the weight of an edge not adjacent to the one that is contracted remains unchanged;

(b) the weight of the contracted edge is redistributed proportionally to the adjacent edges;

(c) if the contraction gives rise to multiple edges, they are identified and their weights are added together.

If $e$ is the edge that is contracted the resulting graph is denoted by $(G / e, c / e)$.

A weighted graph $\left(G_{1}, c_{1}\right)$ is called a minor of $(G, c)$ if $\left(G_{1}, c_{1}\right)$ can be obtained from $(G, c)$ after a sequence of edge deletions or contractions.

\section{REMARKS.}

(1) If $(G, c)$ is a weighted graph and $e$ is an edge which is not a bridge then $c_{e}(G-e)=c(G)$.

(2) If $(G, c)$ is a weighted graph and $e$ is an edge then $c / e(G / e)=c(G)$.

(3) If $G$ is a graph, $e$ is an edge and $c$ and $\tilde{c}$ are two weight functions such that $(G, c) \sim(G, \tilde{c})$ then $\left(G-e, c_{e}\right) \sim\left(G-e, \tilde{c}_{e}\right)$ and $(G / e, c / e) \sim(G / e, \tilde{c} / e)$. 
This last property allows us to define the notion of minor for -equivalence classes. The subgraph relation for equivalence classes is obviously well defined.

\section{DEFINITION 3.}

(a) Given two weighted graphs $\left(G_{1}, c_{1}\right)$ and $\left(G_{2}, c_{2}\right)$, we say that $\left[\left(G_{1}, c_{1}\right)\right]$ is a minor of $\left[\left(G_{2}, c_{2}\right)\right]$ if $\left(G_{1}, c_{1}\right)$ is equivalent to a minor of $\left(G_{2}, c_{2}\right)$.

(b) Given two weighted graphs $\left(G_{1}, c_{1}\right)$ and $\left(G_{2}, c_{2}\right)$, we say that $\left[\left(G_{1}, c_{1}\right)\right]$ is a subgraph of $\left[\left(G_{2}, c_{2}\right)\right]$ if $\left(G_{1}, c_{1}\right)$ is equivalent to a subgraph of $\left(G_{2}, c_{2}\right)$.

\section{OTHER REMARKS.}

(4) The minor relation is an order relation on the set of equivalence classes of weighted graphs.

(5) The minor relation is not a well-quasi-ordering on the set of equivalence classes of weighted graphs: for example, let $G=K_{3}$, denote by $A, B, C$ the vertices of $G$, and for $k \in \mathbb{N}^{*}$ define $c_{k}: E(G) \rightarrow(0, \infty)$ by $c_{k}(\{A, B\})=c_{k}(\{A, C\})=1$, $c_{k}(\{B, C\})=k$. Then $\left\{\left[\left(G, c_{k}\right)\right]\right\}$ is an infinite antichain.

(6) The equivalence class of a weighted subgraph is not necessarily a minor of the equivalence class of the weighted graph. For example, if $(G, c)$ and $\left(G_{1}, c_{1}\right)$ are the graphs below then $\left[\left(G_{1}, c_{1}\right)\right]$ is a subgraph of $[(G, c)]$ but not a minor.

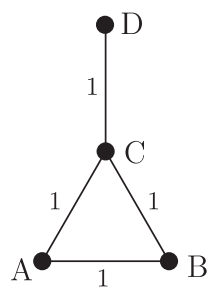

$(G, c)$

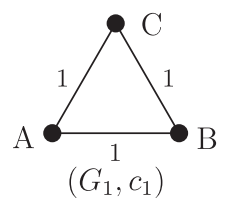

(7) If $(G, c)$ is a weighted graph, $e$ is an edge which is not a bridge and $e_{1}$ and $e_{2}$ are edges adjacent to $e$ then $c_{e}\left(e_{1}\right) /\left(c_{e}\left(e_{2}\right)\right)=c\left(e_{1}\right) /\left(c\left(e_{2}\right)\right)$.

(8) If $(G, c)$ is a weighted graph, $e$ is an edge which will not produce multiple edges by contraction and $e_{1}$ and $e_{2}$ are edges adjacent to $e$ then

$$
c / e\left(e_{1}\right) /\left(c / e\left(e_{2}\right)\right)=c\left(e_{1}\right) /\left(c\left(e_{2}\right)\right) .
$$

Proposition 1. Suppose that $(G, c)$ is a weighted graph. Let $\widehat{G}$ be the cone on $G$, that is, $V(\widehat{G})=V(G) \cup\{X\}$ where $X \notin V(G)$ and $E(\widehat{G})=E(G)$ $\cup \bigcup_{A \in V(G)}\{\{A, X\}\}$. Then there exists $\widehat{c}: E(\widehat{G}) \rightarrow(0, \infty)$ a weight function for $\widehat{G}$ such that:

(a) $[(G, c)]$ is a subgraph of $[(\widehat{G}, \widehat{c})]$;

(b) $[(G, c)]$ is a minor of $[(\widehat{G}, \widehat{c})]$;

(c) $\widehat{c}(\widehat{G})=9 \widehat{c}(G)$. 
Proof. Assume that the vertices of $G$ are $A_{1}, A_{2}, \ldots, A_{n}$. We define the graphs $G_{1}, G_{2}, \ldots, G_{n}$ as follows. For $1 \leq j \leq n: V\left(G_{j}\right)=V(\widehat{G})=\left\{X, A_{1}, \ldots, A_{n}\right\}$ and $E\left(G_{j}\right)=E(G) \cup\left\{\left\{X, A_{1}\right\}, \ldots,\left\{X, A_{j}\right\}\right\}$. Hence $G_{n}=\widehat{G}$. For $j=1,2, \ldots, n$ we shall define inductively the weight $c_{j}$ on $G_{j}$ such that $G$ is a minor of $G_{1}$ and $G_{j}$ is a minor of $G_{j+1}$ for $j=1,2, \ldots, n-1$. We define first $c_{1}$ :

(i) we set $c_{1}\left(\left\{X, A_{1}\right\}\right)=(2 / 3) c\left(A_{1}\right)$;

(ii) if $e$ is an edge of $G$ such that $A_{1}$ is not an end of $e$, we set $c_{1}(e)=c(e)$;

(iii) if $A_{1}$ is an end of $e$, we set $c_{1}(e)=(1 / 3) c(e)$.

Contracting the edge $\left\{X, A_{1}\right\}$ of $G_{1}$, one obtains the graph $G$. The weight of an edge $e$ which is not an edge at $A_{1}$ remains unchanged, that is, its weight is $c_{1}(e)=c(e)$ and the weight of an edge $e$ at $A_{1}$ will be

$$
\begin{aligned}
c_{1}(e)+\frac{c_{1}(e) c_{1}\left(\left\{X, A_{1}\right\}\right)}{c_{1}\left(A_{1}\right)-c_{1}\left(\left\{X, A_{1}\right\}\right)} & =\frac{1}{3} c(e)+\frac{(1 / 3) c(e)(2 / 3) c\left(A_{1}\right)}{c\left(A_{1}\right)-(2 / 3) c\left(A_{1}\right)} \\
& =\frac{1}{3} c(e)+\frac{2}{3} c(e)=c(e) .
\end{aligned}
$$

This means that $(G, c)$ is a minor of $\left(G_{1}, c_{1}\right)$.

We assume now that we have defined the weights $c_{1}, \ldots, c_{j}$ and we shall define $c_{j+1}$ :

(i) $c_{j+1}\left(\left\{X, A_{j+1}\right\}\right)=(2 / 3)\left(c_{j}\left(A_{j+1}+c_{j}(X)\right)\right.$;

(ii) if the edge $e$ is not adjacent to $\left\{X, A_{j+1}\right\}$ then $c_{j+1}(e)=c_{j}(e)$;

(iii) if $e$ is adjacent to $\left\{X, A_{j+1}\right\}$ then $c_{j+1}(e)=(1 / 3) c_{j}(e)$.

A computation similar to the one above shows that deleting $\left\{X, A_{j+1}\right\}$ one obtains $\left(G_{j}, c_{j}\right)$, that is, $\left(G_{j}, c_{j}\right)$ is a minor of $\left(G_{j+1}, c_{j+1}\right)$.

We set $\widehat{c}=c_{n}$. It follows that $[(G, c)]$ is a minor of $[(\widehat{G}, \widehat{c})]$.

It remains to note that when we define $c_{j}$, the weight of an edge of $G$ which is not an edge at $A_{j}$ remains constant and the weight of an edge at $A_{j}$ is multiplied by $1 / 3$. Since an edge has two vertices, its weight in $G_{n}$ will be $1 / 9$ of its initial weight. In other words, for each $e \in E(G), \widehat{c}(e)=(1 / 9) c(e)$. This means that $[(G, c)]$ is a subgraph of $[(\widehat{G}, \widehat{c})]$. At the same time $\widehat{c}(\widehat{G})=c(G)$, hence $\widehat{c}(\widehat{G})=9 \widehat{c}(G)$.

THEOREM 2. For any two connected weighted graphs $\left(G_{1}, c_{1}\right)$ and $\left(G_{2}, c_{2}\right)$ there exists a connected weighted graph $(G, c)$ such that $\left[\left(G_{1}, c_{1}\right)\right]$ and $\left[\left(G_{2}, c_{2}\right)\right]$ are both minors and subgraphs of $[(G, c)]$.

PROOF. Multiplying each weight by a constant (which will not change their equivalence class), we can assume that $c_{1}\left(G_{1}\right)=c_{2}\left(G_{2}\right)=9$. Let us assume that $V\left(G_{1}\right)=\left\{A_{1}, \ldots, A_{s}\right\}$ and $V\left(G_{2}\right)=\left\{B_{1}, \ldots, B_{p}\right\}$. We consider the cones on $G_{1}$ and $G_{2}, V\left(\widehat{G}_{1}\right)=\left\{X_{1}, A_{1}, \ldots, A_{s}\right\}$ and $V\left(\widehat{G}_{2}\right)=\left\{X_{2}, B_{1}, \ldots, B_{p}\right\}$, respectively. We define the weights $\widehat{c}_{1}$ and $\widehat{c}_{2}$ on $\widehat{G}_{1}$ and $\widehat{G}_{2}$ as in the previous proposition. Hence $\widehat{c}_{1}\left(G_{1}\right)=\widehat{c}_{2}\left(G_{2}\right)=1, \widehat{c}_{1}\left(\widehat{G}_{1}\right)=\widehat{c}_{2}\left(\widehat{G}_{2}\right)=9$. Consider the graph $G$ given by: 
(i) $\quad V(G)=V\left(\widehat{G}_{1}\right) \cup V\left(\widehat{G}_{2}\right)$;

(ii) $E(G)=E\left(\widehat{G}_{1}\right) \cup E\left(\widehat{G}_{2}\right) \cup\left\{\left\{X_{1}, X_{2}\right\}\right\}$.

We define a weight $c$ on $G$ as follows:

(i) if $e_{1} \in E\left(G_{1}\right)$, we set $c\left(e_{1}\right):=\widehat{c}_{1}\left(e_{1}\right)$, and if $e_{2} \in E\left(G_{2}\right), c\left(e_{2}\right):=\widehat{c}_{2}\left(e_{2}\right)$;

(ii) $\quad c\left(\left\{X_{1}, A_{i}\right\}\right):=(1 / 8) \widehat{c}_{1}\left(\left\{X_{1}, A_{i}\right\}\right)$ and $c\left(\left\{X_{2}, B_{j}\right\}\right):=(1 / 8) \widehat{c}_{2}\left(\left\{X_{2}, B_{j}\right\}\right)$;

(iii) $c\left(\left\{X_{1}, X_{2}\right\}\right):=5$.

Note that $c\left(\widehat{G}_{1}\right)=c\left(\widehat{G}_{2}\right)=2$ and hence $c(G)=9$. It is clear that $\left[\left(G_{1}, c_{1}\right)\right]$ and $\left[\left(G_{2}, c_{2}\right)\right]$ are subgraphs of $[(G, c)]$. It remains to be checked that they are also minors. We shall show this only for $G_{1}$, the proof for $G_{2}$ being obviously the same.

We contract one by one all edges of $\widehat{G}_{2}$. This will have no effect on the weight of $\widehat{G}_{1}$. As the total weight remains unchanged, at the end of the process, the weight of $\left\{X_{1}, X_{2}\right\}$ will be $5+c\left(\widehat{G}_{2}\right)=7$. Then, if we contract the edge $\left\{X_{1}, X_{2}\right\}$ we obtain $\widehat{G}_{1}$. The weight of an edge of $G_{1}$ does not change. Since the sum of the weights of all edges adjacent to $\left\{X_{1}, X_{2}\right\}$ is $(1 / 8) \widehat{c}_{1}\left(X_{1}\right)=1$, the weight of an edge $\left\{X_{1}, A_{j}\right\}$ will be

$$
\begin{aligned}
c\left(\left\{X_{1}, A_{j}\right\}\right)+\frac{c\left(\left\{X_{1}, A_{j}\right\}\right) c\left(\left\{X_{1}, X_{2}\right\}\right)}{1} & =\frac{1}{8} c_{1}\left(\left\{X_{1}, A_{j}\right\}\right)+\frac{(1 / 8) c_{1}\left(\left\{X_{1}, A_{j}\right\}\right) \cdot 7}{1} \\
& =c_{1}\left(\left\{X_{1}, A_{j}\right\}\right) .
\end{aligned}
$$

Therefore, contracting $\left\{X_{1}, X_{2}\right\}$, we obtain exactly $\left(\widehat{G}_{1}, \widehat{c}_{1}\right)$. In other words, $\left(\widehat{G}_{1}, \widehat{c}_{1}\right)$ is a minor of $(G, c)$. As $\left(G_{1}, c_{1}\right)$ is a minor of $\left(\widehat{G}_{1}, \widehat{c}_{1}\right)$, it follows that $\left(G_{1}, c_{1}\right)$ is a minor of $(G, c)$.

Next we shall introduce another operation for weighted graphs. As before (see Definition 2), this operation is a familiar one for (non-weighted) graphs.

(3) Subdividing an edge by a new node. This means that given a weighted graph $(G, c)$, an edge $\{A, B\}$ of $G$ and a number $k \in(0,1)$, we define the weighted graph $\left(G_{A, B, k}, c_{A, B, k}\right)$ by: $V\left(G_{A, B, k}\right)=V(G) \cup\{X\}$ where $X \notin V(G), E\left(G_{A, B, k}\right)$ $=(E(G) \backslash\{\{A, B\}\}) \cup\{\{A, X\},\{X, B\}\}, c_{A, B, k}(e)=c(e)$ if $e \in E(G) \backslash\{\{A, B\}\}$ and $c_{A, B, k}(\{A, X\})=k c(\{A, B\}), c_{A, B, k}(\{B, X\}=(1-k) c(\{A, B\})$.

A natural question then arises as follows: given a graph $G$ and $c$ and $d$, two weights on $G$, is it possible to perform a sequence of operations of type (3) on $(G, c)$ such that, denoting by $(\tilde{G}, \tilde{c})$ the resulting graph, $[(G, d)]$ is a minor of $[(\tilde{G}, \tilde{c})]$ ? And, if the answer is yes, what is the minimum number of edge subdivisions that is needed?

It turns out that the answer is no, as the following example shows:
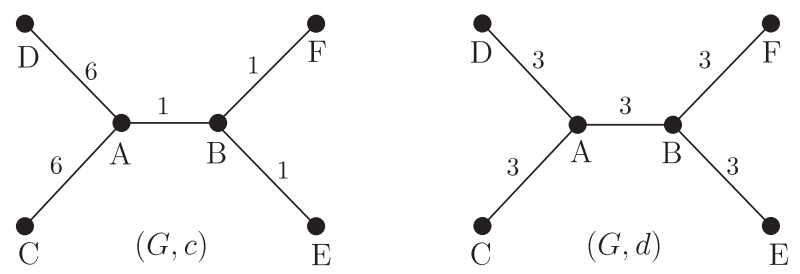
Indeed, given a vertex $A$ of a graph $G$ let us call an $A$-straight path to a terminal vertex a path of the form $A A_{1} \ldots A_{n}$ where $A_{n}$ is a terminal vertex and each $A_{j}$, for $1 \leq j \leq n-1$, has degree 2. If $(G, c)$ is a weighted graph, we denote by $s \operatorname{deg}_{c}(A)$ the sum of total weights of all $A$-straight paths to a terminal vertex and all edges at $A$ that are not part of a $A$-straight path to a terminal vertex. Note that if $e$ is a edge of $G$ then $s \operatorname{deg}_{c}(A) \leq s \operatorname{deg}_{c / e}(A)$. (If $e$ is of the form $\{A, B\}$, the vertex obtained by the identification of $A$ and $B$ is still denoted by $A$.)

For the above example, suppose that $(\tilde{G}, \tilde{c})$ is a graph obtained from $(G, c)$ using a finite number of edge subdivisions. It is clear that $s \operatorname{deg}_{\tilde{c}}(A) \geq 12$.

If $H$ is any tree and $f$ is an edge of $H$ then $H / f$ is also a tree. Since $\tilde{G}$ is a tree, when defining minors of $(\tilde{G}, \tilde{c})$, deleting an edge is not an allowed operation (according to our definition). Suppose that $\left(G, c_{1}\right)$ is a minor of $(\tilde{G}, \tilde{c})$. It follows that $\left(G, c_{1}\right)$ is obtained from $(\tilde{G}, \tilde{c})$ using a sequence of edge contractions. Note that the only two vertices of $\tilde{G}$ of degree at least 3 are $A$ and $B$. This implies that none of these contractions will identify $A$ and $B$. It follows that $s \operatorname{deg}_{c_{1}}(A) \geq 12>9=s \operatorname{deg}_{d}(A)$. In other words, $(G, d)$ cannot be a minor of $(\tilde{G}, \tilde{c})$.

On the positive side, we can show that there exists a sequence of edge subdivisions and edge contractions that will transform $(G, c)$ in $(G, d)$ provided that $c(G)=d(G)$. Moreover, this can be done using at most $|E(G)|-1$ edge subdivisions.

Notation. For a graph $G$, we denote by $r d(G)$ the number $2|E(G)|-|V(G)|$ $=\sum_{v \in V(G)}(\operatorname{deg}(v)-1)$.

By a terminal vertex we understand a vertex of degree 1 .

Definition 4. Suppose that $G$ is a graph, $A \in V(G)$ and $\left\{A, B_{1}\right\}, \ldots,\left\{A, B_{k}\right\}$ are the edges at $A$. We call the blow-up of $G$ at $A$, the graph $\tilde{G}_{A}$ defined as follows:

(i) $\quad V\left(\tilde{G}_{A}\right)=(V(G) \backslash\{A\}) \cup\left\{A_{1}, \ldots, A_{k}\right\}$ where $A_{1}, A_{2}, \ldots, A_{k} \notin V(G)$;

(ii) $E\left(\tilde{G}_{A}\right)=\left(E(G) \backslash\left\{\left\{A, B_{1}\right\}, \ldots,\left\{A, B_{k}\right\}\right\}\right) \cup\left\{\left\{A_{1}, B_{1}\right\}, \ldots,\left\{A_{k}, B_{k}\right\}\right\}$.

\section{REMARKS.}

(1) If $A$ is a vertex of $G$ with $\operatorname{deg}(A)=k$, then $r d\left(\tilde{G}_{A}\right)=r d(G)-(k-1)$.

(2) A weight on $G$ induces a weight on $\tilde{G}_{A}$ and vice versa (simply put $c\left(\left\{A_{j}, B_{j}\right\}\right)$ $\left.=c\left(\left\{A, B_{j}\right\}\right)\right)$.

Lemma 3. Suppose that $G$ is a graph such that $V(G)=\left\{X, A_{1}, \ldots, A_{n}\right\}$ and $E(G)=\left\{\left\{X, A_{1}\right\}, \ldots,\left\{X, A_{n}\right\}\right\}$, and $c$ and $d$ are two weight functions on $G$ such that $c(G)=d(G)$. Then there exists a sequence of edge subdivisions and edge contractions that will transform $(G, c)$ into $(G, d)$. Moreover, this can be done using at most $n-1$ edge subdivisions and the edge contractions will not involve the terminal vertices $A_{1}, A_{2}, \ldots, A_{n}$. 
PROOF. We begin by introducing notation. If $H$ is a graph with $E(H)$ $=\left\{e_{1}, \ldots, e_{p}\right\}$ and $u$ and $v$ two weight functions on $H$ such that $\left(u\left(e_{1}\right) / v\left(e_{1}\right)\right)=\cdots$ $=\left(u\left(e_{k}\right) / v\left(e_{k}\right)\right)<\left(u\left(e_{j}\right) / v\left(e_{j}\right)\right)$ for every $j>k$, we set $m(u, v):=n-k$. Note that $0 \leq m(u, v) \leq n-1$.

We shall prove by induction on $m(c, d)$ that we can transform $c$ into $d$ using at most $m(c, d)$ edge subdivisions and the contractions do not involve terminal vertices.

If $m(c, d)=0$, that is, all ratios $c_{j} / d_{j}$ are equal, since $\sum c_{j}=\sum d_{j}$ it follows that $c_{j}=d_{j}$ for all $j$ and there is nothing to prove.

We assume that the statement is true for $m(c, d)=p-1$ and we prove it for $m(c, d)=p$. Let $k=n-p$, put $c\left(\left\{X, A_{i}\right\}\right)=c_{i}$ and $d\left(\left\{X, A_{i}\right\}\right)=d_{i}$ and assume that $\left(c_{1} / d_{1}\right)=\left(c_{2} / d_{2}\right)=\cdots=\left(c_{k} / d_{k}\right)<\left(c_{k+1} / d_{k+1}\right) \leq\left(c_{k+2} / d_{k+2}\right) \leq\left(c_{n} / d_{n}\right)$.

We add the vertex $P_{k+1}$ on the edge $\left\{X, A_{k+1}\right\}$ such that

$$
c\left(\left\{A_{k+1}, P_{k+1}\right\}\right)=c_{1} \cdot d_{k+1} /\left(d_{1}\right), c\left(\left\{X, P_{k+1}\right\}\right)=c_{k+1}-c_{1} \cdot d_{k+1} /\left(d_{1}\right) .
$$

Note that since $\left(c_{1} / d_{1}\right)<\left(c_{k+1} / d_{k+1}\right)$ the weight of $\left\{X, P_{k+1}\right\}$ will be positive. We denote by $\tilde{c}$ the weight obtained after the contraction of $\left\{X, P_{j}\right\}$. Since this contraction will not create multiple edges, the ratios of those edges that are adjacent to $\left\{X, P_{k+1}\right\}$ will not change. The vertex obtained by identifying $X$ and $P_{k+1}$ will be denoted by $X$ as well. Hence:

(a) for $j \leq k,\left(\tilde{c}\left(\left\{X, A_{j}\right\}\right) / \tilde{c}\left(\left\{X, A_{1}\right\}\right)\right)=\left(c_{j} / c_{1}\right)=\left(d_{j} / d_{1}\right)$;

(b) $\quad\left(\tilde{c}\left(\left\{X, A_{k+1}\right\}\right) / \tilde{c}\left(\left\{X, A_{1}\right\}\right)\right)=\left(c\left(\left\{A_{1}, P_{1}\right\}\right) / c_{1}\right)=\left(c_{1} \cdot d_{k+1} / d_{1}\right) \cdot\left(1 / c_{1}\right)$ $=\left(d_{k+1} / d_{1}\right)$

(c) for $j>s>k+1$, from $\left(\tilde{c}\left(\left\{X, A_{j}\right\}\right) / \tilde{c}\left(\left\{X, A_{1}\right\}\right)\right)=\left(c_{j} / c_{1}\right) \quad$ and $\left(\tilde{c}\left(\left\{X, A_{s}\right\}\right) / \tilde{c}\left(\left\{X, A_{1}\right\}\right)\right)=\left(c_{s} / c_{1}\right) \quad$ we deduce that $\left(\tilde{c}\left(\left\{X, A_{j}\right\}\right) / d_{j}\right)$ $\geq\left(\tilde{c}\left(\left\{X, A_{s}\right\}\right) / d_{S}\right)>\left(\tilde{c}\left(\left\{X, A_{1}\right\}\right) / d_{1}\right)$.

This means that if $\tilde{c}_{j}:=\tilde{c}\left(\left\{X, A_{j}\right\}\right)$ then

$$
\frac{\tilde{c}_{1}}{d_{1}}=\cdots=\frac{\tilde{c}_{k+1}}{d_{k+1}}<\frac{\tilde{c}_{k+2}}{d_{k+2}} \leq \frac{\tilde{c}_{k+3}}{d_{k+3}} \leq \cdots \leq \frac{\tilde{c}_{n}}{d_{n}} .
$$

This shows that $m(\tilde{c}, d)=p-1$ and we can apply the induction hypothesis. As we used only one edge subdivision to transform $c$ into $\tilde{c}$, the proof is complete.

REMARK. In general we cannot transform $c$ into $d$ using $n-2$ or fewer edge subdivisions. For example, if $d_{i}=1$ for all $i$ and $c_{i} \neq c_{j}$ for all $i, j$ with $i \neq j$ and if we use at most $n-2$ edge subdivisions then (at least) two edges are not subdivided. They cannot be contracted either since this will decrease the degree of $X$. It follows that the quotient of their weights will remain constant and it cannot be transformed into 1 .

THEOREM 4. If $G$ is a graph with $n$ edges and $c$ and $d$ are two weights on $G$ such that $c(G)=d(G)$ then there exists a sequence of edge subdivisions and edge contractions 
that will transform $(G, c)$ in $(G, d)$. Moreover, this can be done using at most $n-1$ edge subdivisions.

PROOF. For technical reasons, we shall prove that in fact it is possible to transform $c$ in $d$ such that no contraction will affect a terminal vertex. The proof will be by induction on $\operatorname{rd}(G)$.

If $\operatorname{rd}(G)=0$, as $G$ is connected, $G$ is just a single edge and there is nothing to prove.

Assume that the statement is true for $r d(G) \leq k$ and we shall prove it for $r d(G)=$ $k+1$. We choose a vertex $X$ of $G$ whose degree is at least 2 . Let $A_{1}, \ldots, A_{m}$ be the neighbours of $X$. Let $\tilde{G}_{X}$ be the blow-up of $G$ at $X$ and let $X_{1}, \ldots, X_{m}$ be the new vertices introduced by the blow-up.

Let $G_{1}, G_{2}, \ldots, G_{s}$ be the connected components of $\tilde{G}_{X}$ and let $n_{j}$ be the number of edges of $G_{j}$ (it follows that $\sum n_{j}=n$ ). Note that $r d\left(G_{j}\right) \leq k$. Assume that $c\left(G_{j}\right)>d\left(G_{j}\right)$ for $j \leq q$ and $c\left(G_{j}\right) \leq d\left(G_{j}\right)$ for $q+1<j \leq s$. We shall do the construction in three steps.

Step 1. Let $j \leq q$ be a fixed index and let $\left\{X_{j, 1}, \ldots, X_{j, r}\right\}=V\left(G_{j}\right)$ $\cap\left\{X_{1}, \ldots, X_{m}\right\}$. We define the following weight on $G_{j}$ :

(i) if $e$ is an edge such that $e \cap\left\{X_{j, 1}, \ldots, X_{j, r}\right\}=\emptyset$ then $d_{j}(e)=d(e)$;

(ii) if $e$ is an edge such that $e \cap\left\{X_{j, 1}, \ldots, X_{j, r}\right\} \neq \emptyset$ then $d_{j}(e)=d(e)$ $+\left(c\left(G_{j}\right)-d\left(G_{j}\right)\right) / r$.

Note that since $c\left(G_{j}\right)>d\left(G_{j}\right)$, the weights $d_{j}(e)$ defined as such are positive and $d_{j}\left(G_{j}\right)=c\left(G_{j}\right)$. We apply the induction hypothesis for $G_{j}$ and the weights $c$ and $d_{j}$ and we deduce that we can transform the weight $c$ into $d_{j}$ by a sequence of edge subdivisions and edge contractions, using at most $n_{j}-1$ edge subdivisions and such that the contractions will not involve the terminal vertices of $G_{j}$. In particular, they will not involve $X_{j, 1}, \ldots, X_{j, r}$.

This last condition guarantees that we can perform all these operations in the original graph $G$ (with $\left\{A_{j}, X\right\}$ instead of $\left\{A_{j}, X_{j}\right\}$ ) without changing the weight of an edge that is not in the subgraph corresponding to $G_{j}$.

Step 2. After applying the transformations from Step 1 , for $j=1, \ldots, q$, on $(G, c)$ we denote by $\tilde{c}$ the new weight. For each graph $G_{j}$ with $q+1 \leq j \leq s$ we choose an edge $\left\{A_{l_{j}}, X_{j}\right\}$. We partition the edges of $G$ at $X$ into three subsets:

(i) $\mathcal{U}=\bigcup_{j=1}^{q}\left\{\left\{A_{l}, X\right\}: A_{l} \in V\left(G_{j}\right)\right\}$;

(ii) $\mathcal{V}=\left\{\left\{A_{l_{j}}, X_{j}\right\}: j=q+1, \ldots, s\right\}$;

(iii) $\mathcal{W}=\left\{\left\{X, A_{1}\right\},\left\{X, A_{2}\right\}, \ldots,\left\{X, A_{m}\right\}\right\} \backslash(\mathcal{U} \cup \mathcal{V})$. 
Note that at this moment

$$
\begin{aligned}
\sum_{e \in \mathcal{U}} \tilde{c}(e) & =\sum_{e \in \mathcal{U}} d(e)+\sum_{j=1}^{q}\left(c\left(G_{j}\right)-d\left(G_{j}\right)\right) \\
& =\sum_{e \in \mathcal{U}} d(e)+\sum_{j=q+1}^{s}\left(d\left(G_{j}\right)-c\left(G_{j}\right)\right) .
\end{aligned}
$$

We consider the graph $H$ given by $V(H)=\left\{X, A_{1}, \ldots, A_{m}\right\}, E(H)$ $=\left\{\left\{X, A_{1}\right\}, \ldots,\left\{X, A_{m}\right\}\right\}$, and on $H$ we consider two weights, $\tilde{c}$ and $c_{1}$, where $c_{1}$ is defined as follows:

(i) $c_{1}(e)=d(e)$ if $e \in \mathcal{U}$;

(ii) $c_{1}(e)=\tilde{c}(e)$ if $e \in \mathcal{W}$;

(iii) $c_{1}\left(\left\{A_{l_{j}}, X\right\}\right)=\tilde{c}\left(\left\{A_{l_{j}}, X\right\}\right)+d\left(G_{j}\right)-c\left(G_{j}\right)$ for every $j, q+1 \leq j \leq s$.

Note that all these weights are positive numbers and

$$
\begin{aligned}
c_{1}(H) & =\sum_{e \in \mathcal{U}} d(e)+\sum_{e \in \mathcal{V} \cup \mathcal{W}} \tilde{c}(e)+\sum_{j=q+1}^{s}\left(d\left(G_{j}\right)-c\left(G_{j}\right)\right) \\
& =\sum_{e \in \mathcal{U}} \tilde{c}(e)+\sum_{e \in \mathcal{V} \cup \mathcal{W}} \tilde{c}(e)=\tilde{c}(H) .
\end{aligned}
$$

Hence, we can apply Lemma 3 and after a sequence of edge subdivisions and edge contractions we shall transform $\tilde{c}$ into $c_{1}$. We do this using at most $m-1$ edge subdivisions and the contractions will not involve $A_{1}, \ldots, A_{m}$. As before, this guarantees that we can do the same operations in $G$ without changing anything in the rest of the graph.

After this step the weight function of each $G_{j}$ for $j \leq q$ will be exactly $d$, and for all $j>q$ the total weight of $G_{j}$ will be $d\left(G_{j}\right)$.

Step 3. We apply the induction hypothesis for each $G_{j}, q+1 \leq j \leq s$.

What is left to be done now is to notice that during the entire process the contractions did not involve terminal vertices of $G$ (they were terminal vertices of $G_{j}$ as well) and to count the number edge subdivisions that were used.

(i) At Step 1 we used, for each $j \leq q$ at most $n_{j}-1$ edge subdivisions, hence altogether $\sum_{j=1}^{q}\left(n_{j}-1\right)$.

(ii) At Step 2 we used at most $s-1$ edge subdivisions.

(iii) At Step 3 we used, for each $j>q$ at most $n_{j}-1$ edge subdivisions, hence altogether $\sum_{j=q+1}^{s}\left(n_{j}-1\right)$.

Adding everything together, we used at most

$$
\sum_{j=1}^{s}\left(n_{j}-1\right)+s-1=\sum_{j=1}^{s} n_{j}-1=n-1
$$

edge subdivisions. 


\section{References}

[1] L. Lovász, 'Graph minor theory', Bull. Amer. Math. Soc. (N.S.) 43(1) (2006), 75-86.

[2] N. Robertson and P. D. Seymour, 'Graph minors. XX. Wagner's conjecture', J. Combin. Theory Ser. B 92(2) (2004), 325-357.

CEZAR JOIŢA, Institute of Mathematics of the Romanian Academy, PO Box 1-764, Bucharest 014700, Romania

e-mail: Cezar.Joita@imar.ro

DANIELA JOIŢA, Titu Maiorescu University, Calea Vacaresti nr. 187, sector 4, Bucharest 040056, Romania

e-mail: danielajoita@gmail.com 